\title{
Role of Bronchoalveolar Lavage in the Diagnosis of Sputum Smear Negative Tuberculosis
}

\section{Sandeep Gupta ${ }^{1 *}$ and Kanu Goel ${ }^{2}$}

${ }^{1}$ Consultant Pulmonary and Critical care, Department of Pulmonary Medicine and Critical care, Amar Hospital, Patiala, India ${ }^{2}$ Consultant Pathologist, Department of Pathology, Columbia Asia Hospital, Patiala, India

\begin{abstract}
Mycobacterium tuberculosis is the leading killer of adults in developing and underdeveloped nations. HIV/ AIDS have also contributed to increase in the incidence of tuberculosis. In our study, we evaluated the significance of bronchoalveolar lavage (BAL) in the early diagnosis of occult sputum smear negative pulmonary tuberculosis. We could establish the diagnosis of tuberculosis in $20(87 \%)$ of the sputum smear negative samples with the help of BAL samples. Thus, BAL samples are very useful in early sputum smear negative pulmonary tuberculosis with minimal complications in the hands of an expert.
\end{abstract}

Keywords: Bronchoalveolar lavage; Fibreoptic bronchoscopy; Occult tuberculosis

Abbreviations: BAL: Bronchoalveolar Lavage; PTB: Pulmonary Tuberculosis; TB: Tuberculosis; AFB: Acid Fast Bacilli; ZN Stain: Ziehl Neelsen Stain; LJ Slant: Lowenstein Jensen Slant; TBB: Transbronchial Biopsy

\section{Introduction}

Pulmonary tuberculosis (PTB) remains one of the most important health problems in the world. The World Health Organization (WHO) estimated 9.2 million new cases of tuberculosis (TB) in 2006 (139 per 100000 population), including 4.1 million new smear- positive cases ( $44 \%$ of the total) and 0.7 million HIV positive cases ( $8 \%$ of the total) worldwide [1]. The WHO recommends the detection of acid fast bacilli (AFB) in respiratory specimens as the initial approach to the diagnosis of PTB [2]. However many patients with suspected PTB do not produce sputum spontaneously or are smear-negative for AFB. Thus the diagnosis of TB in these patients is difficult, and in most cases they are treated empirically on the basis of clinical and radiographic findings. This empiric therapy may result in toxicity, increased drug resistance and delay in diagnosis and treatment of conditions other than TB when present. Bronchoscopy is more invasive and expensive than sputum examination, but can provide specimens from specific sites in the lung. Therefore, BAL plays an important role in patients with occult tuberculosis or other mimicking conditions.

Several studies have compared the usefulness of different samples for arriving at an early diagnosis. This study was taken at a community hospital to evaluate the significance of bronchoalveolar lavage culture and acid fast staining as compared to sputum culture and staining for the diagnosis of pulmonary tuberculosis.

\section{Materials and Methods}

Our study design included 23 patients who were negative for AFB in sputum smears but were suspected of having TB clinically or radiographically. The study was conducted over a period of one year from January 2011 to December 2011. Fibreoptic bronchoscopy was performed in these sputum smear negative patients after three consecutive sputum samples to obtain BAL samples along with biopsy wherever possible. Written informed consent was taken from these patients. BAL samples were centrifuged at about $3000 \mathrm{rpm}$ for 15 minutes and smears were prepared from the sediment. Smears were fixed and stained with Ziehl Neelsen (ZN) staining. After air-drying, the smears were examined under oil immersion lens. Cultures were also done on Lowenstein Jensen (LJ) slants and incubated at $37^{\circ} \mathrm{C}$ and screened for any growth at regular intervals two times a week. Cultures were considered negative for acid fast bacteria if no growth was observed after incubation after incubation of LJ slants for a period of 6 weeks. Any growth on the slants was further confirmed by ZN staining.

\section{Results}

There was no significant difference in X-ray and CT findings observed among the smear positive and smear negative patients. Of the 23 patients, 13 (56.5\%) were positive for AFB on staining of BAL samples. Culture was positive in $18(78.3 \%)$ of the BAL samples. Of the BAL smear positive samples, culture was pitive in $10(76.9 \%)$ samples. Sputum culture was positive in $2(8.6 \%)$ patients only. Biopsy was performed in six patients of whom only one was positive for tuberculosis and no other pathology was observed. This patient's BAL was positive for AFB. One biopsy report was positive for squamous cell carcinoma, one for small cell carcinoma of the lung and the other three showed reactive inflammatory changes. Thus the diagnosis of TB could be established in 20 (87\%) of the sputum smear negative samples with the help of BAL samples. None of the patients had any procedure related complications.

\section{Discussion}

Tuberculosis is a leading health problem worldwide. Although the control of TB has improved dramatically in most industrialized countries during the last century, the disease continues to be a major cause of morbidity and mortality in developing nations [3]. Though clinical presentation and radiographic findings provide an important clue for pulmonary tuberculosis, only acid fast stain positivity and culture isolation can provide the definitive diagnosis. Patients with positive tuberculin test and abnormal chest radiographs compatible with tuberculosis pose diagnostic problems and therapeutic dilemma (to treat or not to treat for tuberculosis) to chest physicians. Fibreoptic

*Corresponding author: Dr. Sandeep Gupta, 146, Consultant Pulmonary and Critical care, Department of Pulmonary Medicine and Critical care, Amar Hospital, Patiala, India 147001, Tel : 91-988 839 1241; E-mail: dr_ sandygupta@yahoo.com

Received March 16, 2012; Accepted July 23; Published July 25, 2012

Citation: Gupta S, Goel K (2012) Role of Bronchoalveolar Lavage in the Diagnosis of Sputum Smear Negative Tuberculosis. J Pulmon Resp Med S6:002. doi:10.4172/2161-105X.S6-002

Copyright: (C) 2012 Gupta S, et al. This is an open-access article distributed under the terms of the Creative Commons Attribution License, which permits unrestricted use, distribution, and reproduction in any medium, provided the original author and source are credited. 
bronchoscopic studies provide various types of specimens (aspirates, brushes, lavage fluids and biopsies) which may be useful for early diagnosis of sputum smear negative pulmonary tuberculosis [4,5].

Studies of bronchoscopy have shown that the diagnosis of tuberculosis was increased in cases with sputum smear negative smears using flexible fibreoptic bronchoscopy. Baughman et al. [6] reported $87 \%$ of bronchoscopy sample positivity in sputum smear negative cases. Kennedy et al. [7] observed that early diagnosis of sputum smear negative pulmonary tuberculosis was possible in $38 \%$ of patients if different bronchoscopy procedures such as transbronchial biopsy (TBB) and post-bronchoscopy sputum, in addition to BAL were studied. Pande et al. [8] reported that immediate diagnosis was possible in $35 \%$ of patients using TBB and BAL. Charoenratanakul et al. [9] found that the diagnostic yield of overall bronchoscopic procedures (BAL smear and culture and TBB) was $32.5 \%$ in patients with suspected smear negative pulmonary tuberculosis and Fujii et al. [10] found AFB in $40 \%$ of their bronchoscopy specimens. BAL had significant sensitivity and specificity in a study by Conde et al. [11] and was useful in diagnosis of PTB in $72 \%$ cases. Differences in the results from the various studies may be related to differences in the procedures used, cooperation of the patient, experience of the physician, dose of lignocaine used and transportation of specimens to the laboratory. Among various bronchoscopy specimens, BAL is considered best for the diagnosis of TB.

\section{Conclusion}

To conclude, bronchoscopy should be conducted on all sputum smear negative patients and patients without expectoration, and BAL performance should be a routine procedure as it is simple and usually uncomplicated technique. Most contraindications of bronchoscopy are relative and should be performed whenever the benefits outweigh the risk.

\section{References}

1. WHO report (2008) Global tuberculosis control- surveillance, planning, financing. 393.

2. World Health Organization (2003) Treatment of tuberculosis: Guidelines for National Programmes. (3rdedn), Switzerland, Geneva: WHO.

3. Dye C, Watt CJ, Bleed DM, Hosseini SM, Raviglione MC (2005) Evolution of tuberculosis control and prospects for reducing tuberculosis incidence, prevalence and deaths globally. JAMA 293: 2767-2775.

4. Dolin PJ, Raviglione MC, Kochi A (1994) Global tuberculosis incidence and mortality during 1990-2000. Bull World Health Organ 72: 213-220.

5. Dhanragir H (1995) The changing spectrum of tuberculosis. Experta Medica.

6. Baughman RP, Dohn MN, Loudon RG, Frame PT (1991) Bronchoscopy with bronchoalveolar lavage in tuberculosis and fungal infections. Chest 99: 92-97.

7. Kennedy DJ, Lewis WP, Barnes PF (1992) Yield of bronchoscopy for the diagnosis of tuberculosis in patients with human immunodeficiency virus infection. Chest 102: 1040-1044.

8. Pande BN, Rajan KE, Jena J, Nema SK, Murali M, et al. (1995) Diagnostic yield from fibreoptic bronchoscopy in sputum negative pulmonary tuberculosis cases. Ind J Tub 42: 207.

9. Charoenratanakul S, Dejsomritrutai W, Chaiprasert A (1995) Diagnostic role of fibreoptic bronchoscopy in suspected smear negative pulmonary tuberculosis. Respir Med 89: 621-623.

10. Fujii H, Ishihara J, Fukaura A, Kashima N, Tazawa H, et al. (1992) Early diagnosis of tuberculosis by fibreoptic bronchoscopy. Tuber Lung Dis 73: $167-$ 169

11. Conde MB, Soares SL, Mello FC, Rezende VM, Almeida LL, et al. (2000) Comparison of sputum induction with fibreoptic bronchoscopy in the diagnosis of tuberculosis: experience at an acquired immune deficiency syndrome reference center in Rio de Janeiro, Brazil. Am J Respir Crit Care Med 162: 2238-2240.
This article was originally published in a special issue, Tuberculosis handled by Editor(s). Dr. John L. Ho,Center for Global Health, Centers for Disease Control and Prevention, Haiti 